\title{
LINGUÍSTICA DE CORPUS COMO INSTRUMENTO DE AVALIACCÃO DE TRADUÇÃO LITERÁRIA
}

\author{
Lourdes Bernardes Gonçalves
}

RESUMO: Pretendemos mostrar, aqui, como a Linguística de Corpus pode contribuir para a avaliação da Tradução Literária. Trata-se de uma metodologia que pode explorar, inclusive pela via estatística, uma grande quantidade de textos, percebendo-lhes padrões de ocorrência (de palavras ou expressões), que poderiam passar despercebidas devido ao próprio tamanho dos textos analisados. Também temos a possibilidade de determinar rapidamente concordâncias, definir palavras-chave, baseando-se em frequências de ocorrência, além de outros procedimentos relevantes para a tarefa de tradução. Para tanto, utilizaremos o programa WordSmith Tools, de Mike Scott (1998, versão 3), aplicando a abordagem da Linguística de Corpus na avaliação do conto "A Mother", um dos quinze contos de Dubliners, de James Joyce (1914), e de duas traduções, uma de Hamilton Trevisan (1964) e outra de José Roberto O'Shea (1992). Entendemos que a avaliação de um texto literário supõe dois momentos; no primeiro, acontece a análise do texto original, na busca de suas características especiais, tanto de forma como de conteúdo. No segundo, dá-se o estudo comparado do texto original e suas traduções. Procederemos dessa forma em nossa avaliação, observando escolhas e soluções dos tradutores, numa perspectiva mais descritiva do que prescritiva, mas também apontando inadequações, na tentativa de perceber suas causas e, se possivel, sugerindo novas possibilidades.

Professora Adjunta da Universidade Federal do Ceará. 
UNITERMOS: linguística de corpus; tradução literária; WordSmith Tools; A Mother; James Joyce.

\begin{abstract}
Our purpose here is to show how Corpus Linguistics may contribute to the evaluation of Literary Translation. It is a methodology which can explore a large quantity of texts statistically, identifying occurence patterns of words or phrases - which might go undetected due to the sheer size of the analysed text - quickly determine concords, define keywords, based on occurence frequency, besides other procedures which will be shown. In order to do so, Mike Scott's WordSmith Software (version 3, 1998) will be used, and the Corpus Linguistic approach will be applied in the evaluation of James Joyce's "A Mother", one of his fifteen short stories in Dubliners (1914) and two of its translations into Portuguese, one by Hamilton Trevisan (1964) and the other by José Roberto O'Shea (1992). We consider that the evaluation of a literary text has two distinct moments; one the first, we analyse the original text, observing its special features, either in form or content. In the second, we compare the original to its translations. We will proceed thus here, observing the translators' choices and solutions, in a descriptive perspective, rather than prescriptive, but also pointing out discrepancies, trying to explain its causes and, whenever possible, offering new possibilities.
\end{abstract}

KEYWORDS: corpus linguistics; literary translation; WordSmith Tools; A Mother; James Joyce.

\title{
1 Introdução
}

A Linguística de Corpus consiste basicamente no tratamento de textos em formato eletrônico, por meio de ferramentas computacionais, com vistas aos mais diferentes estudos linguísticos. Mais do que uma metodologia, é uma abordagem, uma nova maneira de se estudar o texto: a utilização de um computador nos permite observar uma grande quantidade de dados, o que possibilita a identificação de padrões de ocorrência no texto. A forma de

TRadTerm, 15, 2009, p. 79-100 
manipular os dados obtidos é também nova, devido ao tratamento estatístico e à capacidade de o computador fornecer, em questão de segundos, informações dificeis, ou mesmo impossíveis, de se coletar manualmente de forma acurada, como, por exemplo, todas as ocorrências de uma palavra com seus contextos, ou a listagem das palavras distintas de um texto por ordem de frequência, entre outras informações.

Softwares especiais, que oferecem ferramentas potentes de análise de corpus, - tais como o WordSmith Tools ou o ParaConc-, vêm sendo desenvolvidos para facilitar o manuseio de grandes corpora, o que é essencial para o pesquisador obter facilmente as informações de que precisa. Palavras podem ser selecionadas dentre milhares, sem a necessidade de ler todos os textos com que se está trabalhando; ocorrências de palavras são fornecidas imediatamente, em termos de percentagens e contextualizadas em linhas de concordância (cf., por exemplo, Sardinha, 2004).

O objetivo deste artigo será justamente demonstrar como essa abordagem pode enriquecer a análise do texto literário e ajudar na avaliação/descrição de suas possíveis traduções. No caso desta pesquisa, foi importante usar tal metodologia para identificar palavras-chave dos textos estudados, isto é, palavras usadas com frequências significativamente maiores do que nos textos de referência. Também buscamos determinar o número de ocorrências de algumas palavras consideradas centrais para a interpretação do texto, examinar certas concordâncias, para avaliar-lhes as prosódias e contextos, além de outros procedimentos sempre enriquecedores para a análise.

O texto que selecionamos como nosso objeto de estudo foi "A Mother", um dos quinze contos de Dubliners, de James Joyce (1914), e duas traduções para o português do Brasil, uma de Hamilton Trevisan (1964) e outra de José Roberto O’Shea (1992). Trata-se, portanto, na linguagem da Linguística de Corpus, de um corpus de estudo paralelo, por serem textos original e suas traduções. As ferramentas de análise utilizadas foram as do programa WordSmith Tools, de Mike Scott (1998, versão 3).

Por compreendermos que a análise de uma tradução literária supõe duas etapas essenciais, dividimos nosso trabalho em função delas, quais sejam: primeiramente uma análise do texto

TradTerm, 15, 2009, p. 79-100 
original, marcando o que há de mais característico nele e/ou os aspectos que não podem ser apagados nas traduções e, em seguida, o estudo das traduções.

De fato, a avaliação da tradução literária supõe dois momentos de análise. No primeiro, o pesquisador se volta para o texto original (TO), observando-lhe as linhas temáticas e estilísticas e qualquer outra característica marcante, traços que devem ser preservados no texto traduzido, na medida do possivel. A segunda etapa consiste em observar o texto traduzido (TT) e contrapô-lo ao TO, a fim de verificar escolhas e soluções, além de possiveis problemas ou inadequações ocorridos no processo tradutório.

\section{A análise do texto original}

Em "A Mother", vemos Mrs. Kearney, mãe dedicada, defendendo furiosamente os direitos da filha. Havia combinado com Mr. Holohan, secretário de uma sociedade musical, que a filha faria quatro apresentações de piano, acompanhando outros artistas. Quando um dos concertos foi cancelado, a senhora exigiu o pagamento integral das apresentações, conforme o combinado. Sentiu-se ainda mais lesada por ter ajudado a organizar os espetáculos. Essa sua conduta mesquinha foi ressaltada através da reação negativa dos outros personagens, o que veio a prejudicar definitivamente a carreira musical da filha.

Uma maneira de perceber as palavras mais importantes no conto é determinar suas palavras-chave. Usando o programa WordSmith Tools, e mais especificamente a ferramenta KeyWord, é possivel definir uma lista de palavras-chave a partir da comparação do TO (corpus de estudo) com um corpus de referência, compilado segundo as especificações do pesquisador. Além da importância dessas palavras na determinação das linhas temáticas, a tradução das palavras-chave assim levantadas será, naturalmente, devido à sua relevância, objeto central de análise na avaliação da tradução.

Também as concordâncias dessas palavras poderão ser obtidas e estudadas com a ferramenta Concord, propiciando a constatação de prosódias, a serem comparadas com as das palavras correspondentes no TT. Para o levantamento das palavras-

TRADTERM, 15, 2009, p. 79-100 
chave, vamos trabalhar com um corpus de estudo, no caso "A Mother", de 4.603 palavras (ou tokens) e dois corpora de referência, com os quais vamos comparar nosso conto.

A fim de que não haja interferências indevidas, a compilação desses corpora de referência deve ser feita criteriosamente. Os contos no início do século XX guardavam ainda uma estrutura bastante rígida, herança da preocupação em diferençar o que se chamava na Inglaterra tale de short-story (May, 1994:44-55 e 165-181). Este último gênero era caracterizado, segundo a definição tradicional, como geralmente apresentando um só enredo, em que trechos dissertativos e mesmo descrições eram reduzidos a um mínimo, exibindo grande compressão de linguagem. Depois da poesia, o conto era considerado o gênero mais compacto. Todos os detalhes irrelevantes à trama eram suprimidos, os personagens se revelavam pelas ações e falas. Assim, faz sentido que o nosso primeiro corpus de referência (refcor), para ser comparado aos contos de Joyce, fosse um corpus de contos. Desse modo, todas as características próprias a todos os contos seriam canceladas na comparação.

A necessidade de que as idiossincrasias próprias da linguagem de um tempo fossem anuladas fez com que escolhêssemos escritores da mesma época de Joyce (1882-1941), ou seja, o início de século XX. Naturalmente, os autores escolhidos não eram a única opção possivel, mas alguns critérios foram considerados para que se criasse um corpus de referência verdadeiramente relevante. Procuramos nomes que influenciaram os rumos do conto como forma literária. Virginia Woolf (1882-1941), nossa primeira escolha, é uma figura reconhecidamente diferenciada na literatura, que se impõe de imediato por muitas razões, inclusive por ter utilizado técnica semelhante à do fluxo da consciência, com os monólogos interiores. Katherine Mansfield (1888-1923), a segunda escolha, além de voluntariamente expatriada e falante nativa não-britânica como Joyce (era neozelandesa), é notadamente uma grande contista. Seguindo a tradição de Tchekhov, elaborou contos perfeitamente dentro da estrutura formal e ainda assim trouxe inovações, como, por exemplo, iniciar o conto in media res, colocar em foco a trivialidade da comédia da vida e algumas vezes deixar o final em aberto, suscitando a direta participação do leitor no enredo. Finalmente, o

TRadTerm, 15, 2009, p. 79-100 
terceiro, D. H. Lawrence (1885-1930), aparece como um contista relevante e polêmico, embora não se possa dizer que considerava o efeito de compressão tão essencial como as escritoras mencionadas. Desafiou as convenções de sua época e privou, assim como Mansfield e Woolf, dos círculos de escritores mais seletos de seu tempo. Dessa forma, o nosso primeiro corpus de referência foi então composto como mostra a Figura 1:

\begin{tabular}{|c|c|c|}
\hline Autores & $\mathbf{N}^{\mathbf{0}}$ de contos & Total de palavras \\
\hline V. Woolf & 23 & 53.659 \\
\hline K. Mansfield & 12 & 79.087 \\
\hline D. H. Lawrence & 17 & 79.845 \\
\hline TOTAL & $\mathbf{5 2}$ & $\mathbf{2 1 2 . 5 9 1}$ \\
\hline
\end{tabular}

Fig. 1: Composição do refcor

Note-se que os contos de Virginia Woolf, embora em maior número, constituem o menor sub-corpus. Porém, conforme atesta Margaret Drabble (1996), editora do The Oxford Companion to English Literature, esses foram todos os contos que Woolf escreveu.

Com o auxílio das ferramentas mencionadas, fizemos a lista de palavras-chave entre "A Mother" e o refcor. Para que se avalie a importância de uma palavra-chave, foi introduzido o conceito de keyness, que fornece um número para se medir "quão" chave a palavra é no corpus de estudo. Essa variável, traduzida por "chavicidade", dá a medida em que uma palavra é original e característica do corpus de estudo, em relação ao corpus de referência. Trata-se de um resultado estatístico obtido quando se comparam as WordLists dos dois corpora (utilizando-se as fórmulas estatísticas do qui-quadrado ou log-likelihood) (Sardinha, 2004:104). Associada a esse número, há uma grandeza estatística selecionada pelo usuário, $p$, que dará a significância do resultado obtido. Assim, por exemplo, no caso de se usar o teste do qui-quadrado, "um valor [de $p$ ] de 0,01 sugere um risco de $1 \%$ de se estar errado ao se afirmar uma relação" (Scott, 1998, WordSmith Tools Manual, tradução nossa). Costuma-se aceitar como chavicidade significativa valores maiores que 4,0 (idem, ibidem).

TradTerm, 15, 2009, p. 79-100 
A título de ilustração, apresentamos, na Figura 2, as dez primeiras palavras-chave da comparação do corpus de referência refcor - com o corpus de estudo - "A Mother" - $(\mathrm{p}=0,001)$, juntamente com as frequências em cada corpus, as respectivas percentagens e a chavicidade (keyness). As colunas mostram, respectivamente, a palavra-chave, sua ocorrência no corpus de estudo, a percentagem que isso representa dentre as palavras diferentes do texto, a frequência da palavra-chave no (refcor), sua percentagem nesse corpus e, finalmente, a chavicidade:

\begin{tabular}{|rrrrrrr|}
\hline $\mathbf{N}$ & WORD & FREQ. & \multicolumn{2}{c}{ TXT \%FREQ. } & R.LST \% KEYNESS \\
1 & KEARNEY & 50 & 1,10 & 0 & & 387,3 \\
2 & HOLOHAN & 37 & 0,81 & 0 & & 286,5 \\
3 & MR & 75 & 1,65 & 173 & 0,08 & 284,6 \\
4 & MRS & 49 & 1,08 & 259 & 0,12 & 120,5 \\
5 & FITZPATRICK & 14 & 0,31 & 0 & & 108,3 \\
6 & CONCERT & 14 & 0,31 & 1 & & 101,0 \\
7 & KATHLEEN & 12 & 0,26 & 0 & & 92,9 \\
8 & HEALY & 11 & 0,24 & 0 & & 85,1 \\
9 & COMMTTEF & 10 & 0,22 & 1 & & 70,7 \\
10 & CONCERTS & 10 & 0,22 & 1 & & 70,7 \\
& & & & & & \\
\hline
\end{tabular}

Fig. 2: Palavras-chave (com refcor)

Dividimos as palavras-chave lexicais (ou de conteúdo), encontradas na lista completa, em áreas semânticas, reunindo palavras cujos significados fariam com que essas palavras se unissem em torno de um assunto, ou interesse. Algumas palavras foram colocadas numa ou noutra área semântica somente depois de estudo cuidadoso das concordâncias associadas a elas. Essas palavras-chave se enquadraram, em sua grande maioria, nos seguintes campos (palavras fornecidas com as respectivas chavicidades entre parênteses):

Área de música: concert $(101,0)$, concerts $(70,7)$, artistes $(69,6)$, baritone $(61,9)$, tenor $(61,9)$, stewards $(38,7)$, accompanist $(38,7)$, hall $(36,6)$, notes (4 oc. dentre 5 - 23,8), audience $(23,4)$, clapping $(23,2)$, item $(23,2)$, items $(23,2)$, music $(22,2)$, bass $(21,5)$, part (6 oc. de $9-20,2)$, interval $(18,8)$, screen $(18,8)$, grand (opera, concert-

TradTerm, 15, 2009, p. 79-100 
$16,0)$, auditorium $(15,5)$, artiste $(15,5)$, intervals $(15,5)$, series $(15,5)$, opera $(15,5)$, contralto $(15,5)$, applauded $(11,7)$, musical $(11,7)$.

Área de negócios: committee $(70,7)$, contract $(63,2)$, appeal $(26,0)$, paid $(24,8)$, notes ( 1 oc. dentre $5-23,8)$, guineas $(23,4)$, secretaries $(23,2)$, secretary $(18,8)$, society $(17,4)$, conduct $(=$ behaviour $-16,6)$, expense $(16,6)$, arguing $(15,5)$, receive $(15,5)$, terms $(15,5)$, advised $(15,5)$, business $(14,5)$, treated $(13,8)$.

Como a música faz parte do enredo, assim como as negociações sobre os termos da apresentação dos concertos, é razoável termos as duas áreas acima privilegiadas em termos semânticos. No caso dos negócios, a Linguística de Corpus fornece uma evidência dos interesses mercenários de Mrs. Kearney, apoiada em dados estatísticos, isto é, no número de ocorrências de palavras associadas com negócios no texto.

Um outro corpus que pode ser usado como referência é a coletânea de contos a que "A Mother" pertence, Dubliners, excluindo-se o próprio conto. Esse corpus denominamos dub-am, com 64.545 palavras.

Constatamos que o corpus de referência $d u b$-am, apesar de bem menor que o refcor, é mais de 14 vezes maior que o nosso corpus de estudo, o que é um tamanho aceitável, como se percebe pela afirmação de Sardinha: "os tamanhos críticos de corpora de referência são 2, 3 e 5 vezes o tamanho do corpus de estudo. Corpora de referência com estas dimensões retornam significativamente mais palavras-chave do que corpora de tamanhos menores" (Sardinha, 2004:102). Essa nova comparação nos revelará o que o conto em estudo tem de específico, dentro da coletânea de Joyce.

Assim, determinamos as palavras-chave geradas pela comparação do conto "A Mother" com esse segundo corpus de referência, como mostra a Figura 3:

TRAdTerm, 15, 2009, p. 79-100 


\begin{tabular}{|rrrrrrr|}
\hline $\mathbf{N}$ & WORD & FREQ. OTHR.TXT \% FREQ. B-AM .LST \%KEYNESS \\
1 & KEARNEY & 50 & 1,10 & 1 & & 261,4 \\
2 & HOLOHAN & 37 & 0,81 & 1 & & 191,4 \\
3 & MRS & 49 & 1,08 & 68 & 0,11 & 115,9 \\
4 & SHE & 115 & 2,53 & 580 & 0,91 & 80,1 \\
5 & FITZPATRICK & 14 & 0,31 & 0 & & 75,8 \\
6 & HEALY & 11 & 0,24 & 0 & & 59,5 \\
7 & KATHLEEN & 12 & 0,26 & 1 & & 58,1 \\
8 & CONCERT & 14 & 0,31 & 4 & & 57,3 \\
9 & CONCERTS & 10 & 0,22 & 1 & & 47,6 \\
10 & DAUGHTER & 16 & 0,35 & 14 & 0,02 & 47,1 \\
& & & & & & \\
\hline
\end{tabular}

Fig.3: palavras-chave (com dub-am)

Ao confrontarmos o nosso conto com $d u b$-am, isto é, com a coleção dos outros contos de Dubliners ( $\mathrm{p}=0,001)$, encontramos os mesmos grupos temáticos, mas agora com um número menor de palavras e com chavicidades mais baixas. Isso indica que a maioria dos outros contos, em maior ou menor grau, lida com música e negócios, uma vez que a comparação com os outros textos de Dubliners também acusou a presença, ainda que menor, de palavras relacionadas à música e a negócios.

Outro aspecto interessante é pesquisar o tratamento dado por Joyce aos personagens. Encontramos no confronto "A Mother" com o refcor. Mr. (290,3), Mrs. (120,7), gentlemen (12,8), Miss (12,2), lady $(11,0)$ e madam $(7,2)$. Esse é um dado revelador do tratamento cerimonioso de Joyce dedicado a seus personagens. Para verificar se é um caso isolado desse conto, podemos observar as palavras-chave geradas pela comparação do conto com o segundo corpus de referência $(d u b-a m)$. Nessas novas palavras-chave encontramos: $M r s$. $(116,2)$, Mr. $(32,0)$, Miss $(14,8)$, madam $(14,3)$ e lady $(13,1)$. Notamos que a chavicidade de $M r$. é bem menor, mostrando que, apesar de o conto ter vários personagens masculinos, os outros contos também têm; e os pronomes de tratamento feminino com maior chavicidade se explicam, porque o conto mostra proporcionalmente maior participação feminina do que a obra Dubliners em geral.

Já Mrs. aparece como chave nas duas listas, com altas chavicidades: na comparação com o refcor, 120,5 e, com o dub$a m, 115,9$. Esses dados são reveladores, porque apontam para o irônico contraste entre o desrespeito completo exibido não só por

TradTerm, 15, 2009, p. 79-100 
Mrs. Kearney, mas também pelos membros do comitê da Eire Abu. Será importante observar como os tradutores lidaram com os pronomes de tratamento.

Ainda em relação a Mrs. Kearney, com a ferramenta Concord, pesquisamos os contextos em que aparecem Mrs. Kearney e she (quando se refere a Mrs. Kearney), observando em especial os verbos usados com os sujeitos. Vejamos:

Área de negócios e argumentação: repeat-asked (3) - boughtsaid (7) - perceived - was taking (note) - spoke - repeated - might have taken (into consideration) - explained - saw (= understand: 1 oc.dentre 3) - determined-learned-was speaking.

Notamos que não há qualquer verbo relativo à apreciação pela música ou exibindo consideração por atividades artísticas por parte de Mrs. Kearney, o que a coloca como insensivel e mercenária dentro da perspectiva joyceana, de que a sensibilidade musical tem uma conotação positiva, no que se refere à personalidade do personagem.

A palavra friends (chavicidade 39,9 em relação ao refcor e 10,3 em relação ao dub-am) é uma palavra sempre interessante de se verificar em Joyce, por causa da conotação irônica que ela muitas vezes assume em suas obras. Verificamos, portanto, as prosódias dessa palavra, como se costuma definir em Linguística de Corpus as conotações, o que é fácil com o uso da ferramenta Concord do WordSmith Tools (Figura 4):

Concordance
N
1 t. However, when she drew near the limit and her friends began to loosen their tongues about her,
2 Irish picture postcards to their friends and these friends sent back other Irish picture postcards. O
3 en as she stood chatting to one of her Nationalist friends, Miss Healy, the contralto. An unknown
4 . They were all friends of the Kearneys - musical friends or Nationalist friends, and, when they ha
$5 \mathrm{lly}$ pale and unbending in manner she made few friends at school. When she came to the age of
$6 \mathrm{~s}$ of the Kearneys - musical friends or Nationalist friends, and, when they had played every little c
7 of concerts. He had a game leg, and for this his friends called him Hoppy Holohan. He walked up
8 and her sister sent Irish picture postcards to their friends and these friends sent back other Irish p
9 kets for the final concert and sent them to those friends who could not be trusted to come otherwi
10 at the corner of Cathedral Street. They were all friends of the Kearneys - musical friends or Natio
11 g out his head and exchanging a laugh with two friends in the corner of the balcony. In the cours

Fig. 4: Concordâncias de friends

TRadTerm, 15, 2009, p. 79-100 
Ao examinarmos as concordâncias, notamos que há prosódias negativas $(1,7,9)$ e que as outras frases se referem a amizades vagas, não muito estreitas, pois não se classificam verdadeiros amigos como "meus amigos musicais" ou "meus amigos nacionalistas". E a frase 5 mostra, com o uso da negativa, que, apesar de a prosódia de friends ser positiva, amigos eram uma ausência na vida escolar da atual Mrs. Kearney.

Será curioso observar as prosódias da palavra "amigos" nas traduções do conto, o que será feito mais adiante.

Como friends apareceu como chave nas duas comparações, resolvemos investigar friend e friendship:

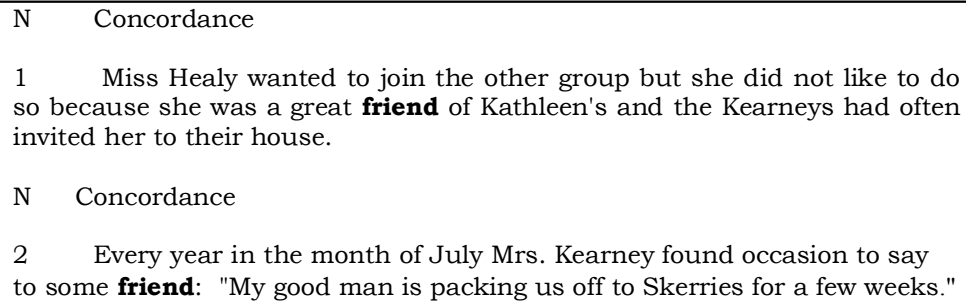

Fig. 5: Concordâncias de friend

Observamos que, na primeira linha de concordância, friend se refere a uma amizade real, uma vez que Miss Healy resolve apoiar a amiga, mesmo sem concordar com a posição que Kathleen foi forçada a tomar. Já no segundo caso, friend ocorre numa situação em que a expressão some friend é claramente usada para conhecidos e não para amigos íntimos.

Não encontramos qualquer ocorrência de friendship no texto "A Mother", porém friendliness aparece uma vez:

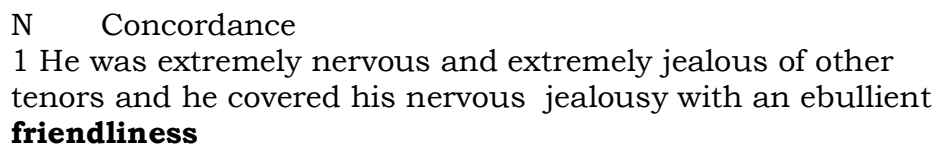

Fig. 6: Concordância de friendliness

Vemos imediatamente a conotação de falsidade que a palavra assume no contexto. Observaremos mais adiante as traduções desse grupo de palavras. 
Até este momento, em nossas observações do texto original, dados objetivos nos mostraram as áreas temáticas mais importantes do conto em termos de percentagem lexical, no caso privilegiando as áreas semânticas de música e negócios. Foi revelado o tratamento cerimonioso dado por Joyce a seus personagens, com o uso de pronomes de tratamento como $M r$. e $M r s$. Pudemos também notar traços da personalidade de Mrs. Kearney e as conotações da palavra friends, friend e friendliness. Na sequência, veremos como esses dados podem ser úteis na avaliação das traduções de "A Mother".

\subsection{Análise comparativa do texto original e traduções}

Iniciamos as observações referentes à comparação do texto original e os textos traduzidos, respectivamente por Hamilton Trevisan e José Robert O'Shea, com uma macroanálise dos três textos em termos estatísticos. Com a ferramenta WordList, podemos obter dados estatísticos sobre o número de parágrafos dos textos, tamanho médio das frases etc., selecionando a lista $\mathrm{S}$ (Statistics), como mostra a Figura 7:

\begin{tabular}{rrrr|}
$\mathbf{N}$ & 1 & 1 & 1 \\
Text File & AMOTHR.TXT & MÃE-HT.TXT & MÃE-OS.TXT \\
Bytes & 25.696 & 24.266 & 26.340 \\
Tokens & 4.602 & 3.953 & 4.394 \\
Types & 428 & 422 & 450 \\
Type/Token Ratio & 9,30 & 10,68 & 10,24 \\
Standardised Type/Token & 41,50 & 49,47 & 48,28 \\
Ave. Word Length & 4,32 & 4,89 & 4,77 \\
Sentences & 231 & 219 & 211 \\
Sent.length & 16,13 & 14,04 & 15,86 \\
sd. Sent. Length & 9,97 & 7,93 & 8,67 \\
Paragraphs & 84 & 83 & 85 \\
Para. length & 54,58 & 47,63 & 51,69 \\
sd. Para. length & 63,09 & 54,71 & 59,22 \\
\hline
\end{tabular}

AMOTHR = "A Mother" de Joyce | MÃE-HT = tradução de Trevisan |

MÃE-OS = tradução de O'Shea

Fig. 7: Dados estatísticos

Uma conclusão que se pode tirar do quadro acima é que o texto original tem mais palavras (tokens) do que os textos traduzidos, o que não é o que intuitivamente se supõe, ainda mais

TradTerm, 15, 2009, p. 79-100 
que a língua inglesa é muitas vezes considerada como mais sucinta que o português.

É curioso notar que o número de frases de Trevisan é menor (219) que o número de frases do original (231), sugerindo que ele uniu algumas frases separadas no texto original. Porém, para surpresa nossa, as frases de Trevisan são mais curtas na média do que as de Joyce $(12,95 \%)$. Isso poderia significar que Trevisan basicamente alongou as frases curtas de Joyce, de modo que se manteve a predominância de frases longas em Joyce.

Mas há dificuldades no programa no que diz respeito à contagem de frases. Por exemplo, a contagem de pontos é usada para definir o número de frases. Assim, erros podem ser introduzidos por causa de pontos que não definam frases, como no caso de $M r$. ou $M r s$. e também das reticências, que seriam contariam como três frases. Contudo, como a diferença entre os números de frases e os tamanhos das frases do texto original e das duas traduções não foi expressiva, abandonamos essa linha de investigação, concluindo que o tamanho das frases, assim como o número de parágrafos, neste caso em particular, não pode ser considerado decisivo para a classificação das traduções como estrangeirizantes ou domesticadoras.

Considerando a lista F (Frequency) fornecida pela ferramenta WordList, podemos fazer algumas observações. Em relação aos (pro)nomes de tratamento como indicadores de formalidade, que foi um fator preponderante para Joyce, lembramos que a palavra de maior chavicidade foi $M r$., na comparação com o refcor. Assim, consideramos importante a verificação de suas traduções e, para tanto, construímos a seguinte tabela (Figura 8):

\begin{tabular}{|c|c|c|c|c|c|}
\hline JJ & oc. & HT & oc. & OS & oc. \\
\hline Mrs. & 49 & - & - & Mrs. & 51 \\
\hline madam & 4 & madame & 4 & madame & 4 \\
\hline lady/ies & 9 & senhora & 39 & senhora & 9 \\
\hline Miss & 18 & senhorita & 14 & Miss & 17 \\
\hline Mr. & 75 & - & - & Mr. & 75 \\
\hline TOTAL & $\mathbf{1 5 5}$ & & $\mathbf{5 7}$ & & $\mathbf{1 5 6}$ \\
\hline
\end{tabular}

Fig. 8: Síntese da tradução dos indicadores de formalidade

TradTerm, 15, 2009, p. 79-100 
Observe-se que lady/'dama' e madam/'madame' foram aqui incluídos por serem também indicadores de formalidade. Um estudo mais detalhado desses indicadores no texto original - realizado pela observação das palavras-chave do conto quando comparado com os outros contos de Joyce $(d u b-a m)$ - revelou que o conto é mais formal do que os outros, considerados em conjunto, uma vez que vários tratamentos formais são chave.

Essa observação tem implicações temáticas, uma vez que torna evidente a ironia entre a formalidade do tratamento e o desrespeito mútuo entre os personagens principais. Ficou evidente a tendência estrangeirizante de O'Shea, ao preservar os pronomes de tratamento em inglês, bem como a tendência domesticadora de Trevisan, ao fazer o contrário. Examinando as ocorrências, notamos em Trevisan uma tendência a eliminar os pronomes de tratamento, introduzindo um grau de informalidade que o distanciou do texto de partida, e apagando, ainda, o efeito irônico; O'Shea permaneceu bem mais próximo do texto original.

É possível, também, utilizando as listas de palavras dos três textos, determinar os substantivos mais frequentes (Figura 9):

\begin{tabular}{|c|c|c|c|c|c|c|}
\hline $\mathbf{n}^{\circ}$ & JoYCE & oc. & TREVISAN & oc. & O'SHEA & oc. \\
\hline 1 & room & 18 & filha & 19 & filha & 17 \\
\hline 2 & daughter & 16 & comitê & 12 & camarim & 12 \\
\hline 3 & concert & 14 & sala & 11 & marido & 12 \\
\hline 4 & friends & 11 & contrato & 9 & sala & 12 \\
\hline 5 & man & 11 & marido & 9 & comitê & 11 \\
\hline 6 & time & 11 & público & 9 & noite & 10 \\
\hline 7 & committee & 10 & tenor & 9 & recitais & 10 \\
\hline 8 & concerts & 10 & baritono & 8 & contrato & 9 \\
\hline 9 & artistes & 9 & concerto & 8 & momento & 9 \\
\hline 10 & contract & 9 & concertos & 8 & recital & 9 \\
\hline
\end{tabular}

Fig. 9: Substantivos mais frequentes nos 3 textos, com o $n^{\circ}$ de ocorrências

Algumas palavras do texto original nos chamam a atenção por não constarem entre os dez substantivos mais ocorrentes nas traduções - friends, man e time-, e isso é um bom ponto de partida para outra investigação. Das três palavras, estudaremos as duas primeiras, a título de demonstração do método, começando com a palavra friends.

TradTerm, 15, 2009, p. 79-100 
Uma opção de análise poderia ser verificar as traduções, mas há a possibilidade de as palavras terem sido utilizadas em outros contextos como forma de compensação. Assim, como exemplo, vejamos, nas Figuras 10 e 11, as concordâncias de "amigos/as". Primeiramente, em Hamilton Trevisan:

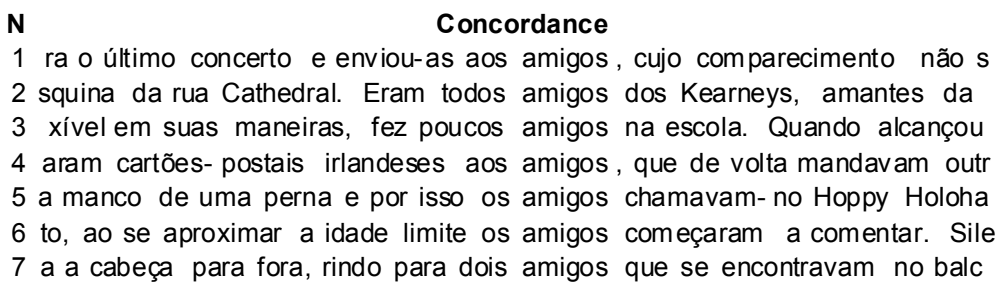

Fig. 10: Concordâncias de amigos - Trevisan

Em seguida, em José Roberto O’Shea:

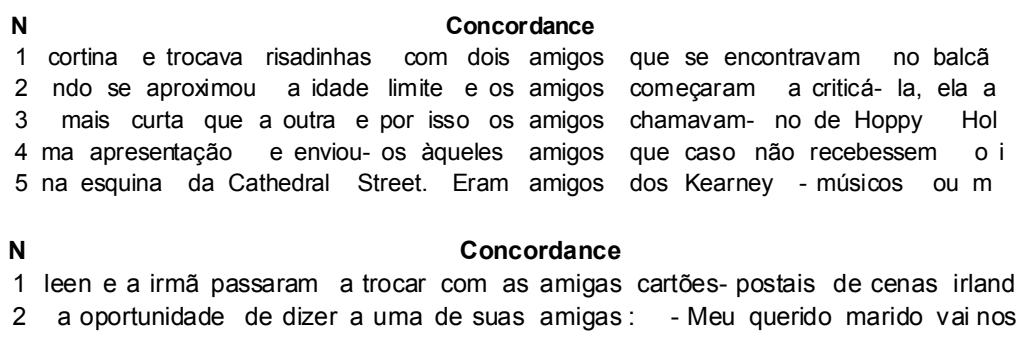

Fig. 11: Concordâncias de amigos/as - O'Shea

Vemos que todas as vezes que "amigos/as" (Trevisan não usou "amigas") foi usado correspondeu a traduções literais de friends, porém nem todas as vezes friends foi traduzido literalmente. $\mathrm{E}$ o fato de que friends foi traduzido por duas formas, feminina e masculina, justifica o fato de não aparecerem na lista dos dez substantivos mais ocorrentes. É interessante verificar como Trevisan traduziu as duas frases em que O'Shea usou a palavra “amigas". Uma tradução é a de número 4, na Figura 10, e a outra corresponde à Figura 12:

TradTerm, 15, 2009, p. 79-100 
N 598 Sentences and headings

$41<$ !--L1, S 21-->Every year in the month of July Mrs. Keamey found occasion to say to some friend:

$42<$---L2, S 21-->Todo ano, no mês de julho, a senhora Keamey arranjava jeito de dizer a algum amigo:

Fig. 12: Tradução da frase S21 por Trevisan

A forma em que o original e sua tradução aparecem na Figura 12 é a forma apresentada pelo programa, na tela do computador, em que L1 representa a língua de partida, L2 a língua de chegada e $\mathrm{S} 21$, por exemplo, a frase de número 21 , considerando-se o título como $\mathrm{S} 1$.

Observe-se que o uso de some friend é vago o bastante para poder valer ou como 'algum amigo' (Trevisan) ou 'uma de suas amigas' (O'Shea); mas, considerando-se que Mrs. Kearney queria 'se gabar', é mais provável que ela contasse o episódio às amigas, o que coloca a tradução de O'Shea mais próxima do texto original.

A palavra man tem outras justificativas para que sua tradução literal não apareça, como veremos nas concordâncias a seguir (Figura13):

\begin{tabular}{|l} 
N Concordance \\
1 e smiled and shook his hand. He was a little man, with a white, vacant face. She notice \\
2 o have treated her like that if she had been a man. But she would see that her daughter \\
3 Id see that it went in. He was a grey-haired man with a plausible voice and careful mann \\
4 ell, the second tenor, was a fair-haired little man who competed every year for prizes at \\
5 occasion to say to some friend: My good man is packing us off to Skerries for a few v \\
6 The bass, Mr Duggan, was a slender young man with a scattered black moustache. He \\
7 e room by instinct. He was a suave, elderly man who balanced his imposing body, wh \\
8 ried life, Mrs Kearney perceived that such a man would wear better than a romantic pers \\
9 ly and the baritone. They were the Freeman man and Mr O'Madden Burke. The Freeman \\
10 man and Mr O'Madden Burke. The Freeman man had come in to say that he could not \\
11 ile Mr Holohan was entertaining the Freeman man Mrs Kearney was speaking so animat
\end{tabular}

Fig. 13: Concordâncias de man

Na frase 5, a expressão "my good man" refere-se a "esposo" ou "marido", e foi traduzida como mostra a Figura 14:

\begin{tabular}{|l|l|}
\hline JJ & "My good man is packing us off to Skerries for a few weeks." \\
\hline HT & - Meu bom esposo vai nos mandar a Skerries por algumas semanas. \\
\hline OS & - Meu querido marido vai nos mandar passar algumas semanas em Skerries. \\
\hline
\end{tabular}

Fig. 14: Traduções da frase de concordância $n^{\circ} 5$

TRadTerm, 15, 2009, p. 79-100 
Cumpre ressaltar que na figura 14 (acima) apresentamos uma montagem do original com as respectivas traduções, que será usada daqui em diante. Ela foi composta a partir do utilitário Viewer \& Aligner, com JJ = Joyce; HT = Trevisan; OS = O'Shea.

Voltemos às concordâncias. As últimas três concordâncias usam man no sentido de jornalista, o que não se usa na língua portuguesa, como mostra o exemplo na Figura 15:

\begin{tabular}{|l|l}
\hline JJ & They were the Freeman man and Mr. O'Madden Burke.
\end{tabular} HT Eram o senhor O'Madden Burke e um repórter do Freeman. OS Um era repórter do Freeman e o outro era Mr. O'Madden Burke.

Fig. 15: Traduções da frase de concordância $n^{\circ} 9$

O número de ocorrências de man, no sentido usual, se reduz então a 7 , o que é inferior às outras ocorrências da tabela de substantivos.

Passamos então à observação da palavra time. As concordâncias (Figura 16) mostram um padrão - from time to time - que justifica de imediato a não ocorrência de "tempo" nas listas de palavras mais frequentes nas traduções:

\begin{tabular}{|l} 
N Concordance \\
1 selves nervously, glanced from time to time at the mirror and $r$ \\
2 at the edge of the screen, from time to time jutting out his hea \\
3 the frontal sinus. From time to time everyone glanced at Mrs \\
4 iffly before her and from time to time changing the direction of $h$ \\
5 nervously, glanced from time to time at the mirror and rolled an \\
6 e-quarters of an hour before the time at which the concert was \\
7 effect on the frontal sinus. From time to time everyone glanced \\
8 th them. She waited until it was time for the second part to beg \\
9 usic stiffly before her and from time to time changing the direc \\
10 dge of the screen, from time to time jutting out his head and e \\
11 the interval, content. All this time the dressing- room was a
\end{tabular}

Fig. 16: Concordâncias de time

Há, na verdade, duas causas prováveis para isso: (1) a expressão não deve ter sido traduzida sempre por outra que contenha "tempo", e (2) a palavra time ocorre duas vezes em cada expressão, fazendo com que cada expressão seja contada duas vezes. E foi realmente isso que ocorreu, como vemos nos exemplos na Figura 17:

TradTerm, 15, 2009, p. 79-100 


\begin{tabular}{|c|l|}
\hline JJ & $\begin{array}{l}\text { Madam Glynn took her stand in a corner of the room, holding a } \\
\text { roll of music stiffly before her and from time to time changing } \\
\text { the direction of her startled gaze. }\end{array}$ \\
\hline HT & $\begin{array}{l}\text { Madame Glynn havia parado num canto da sala, com a partitura } \\
\text { aberta nas mãos, desviando de quando em quando a direção do } \\
\text { seu olhar assustado. }\end{array}$ \\
\hline OS & $\begin{array}{l}\text { Madame Glynn colocou-se no canto da sala, empunhando } \\
\text { firmemente a partitura enrolada e de vez em quando mudando a } \\
\text { direção do seu olhar assustado. }\end{array}$ \\
\hline JJ & $\begin{array}{l}\text { The artistes talked among themselves nervously, glanced from } \\
\text { time to time at the mirror and rolled and unrolled their music. }\end{array}$ \\
\hline HT & $\begin{array}{l}\text { Os artistas conversavam nervosamente, olhando-se de quando } \\
\text { em quando no espelho, enrolando e desenrolando as partituras. }\end{array}$ \\
\hline OS & $\begin{array}{l}\text { Músicos e cantores conversavam um pouco nervosos, olhavam-se } \\
\text { a todo momento no espelho e enrolavam e desenrolavam suas } \\
\text { partituras. }\end{array}$ \\
\hline
\end{tabular}

Fig. 17: Traduções de from time to time

A visualização das frases do texto original, contrapostas aos textos traduzidos, proporcionada pela ferramenta Viewer \& Aligner, facilita uma microanálise de cada frase do texto. Podemos, assim, perceber alguns problemas pontuais com mais facilidade, como nos casos que passamos a apresentar, a título de exemplos.

Há desvios lexicais de várias ordens, desde desvios semânticos ao apagamento de duplos sentidos.

Por exemplo, há alguns casos claros de falta de correspondência de sentido, como os que se apresentam na Figura 18 abaixo:

\begin{tabular}{|c|l|}
\hline JJ & $\begin{array}{l}\text { Madam Glynn took her stand in a corner of the room, } \\
\text { holding a roll of music stiffly before her and from time } \\
\text { to time changing the direction of her startled gaze. }\end{array}$ \\
\hline HT & $\begin{array}{l}\text { Madame Glynn havia parado num canto da sala, com } \\
\text { a partitura aberta nas mãos, desviando de quando em } \\
\text { quando a direção do seu olhar assustado. }\end{array}$ \\
\hline OS & $\begin{array}{l}\text { Madame Glynn colocou-se no canto da sala, } \\
\text { empunhando firmemente a partitura enrolada e de } \\
\text { vez em quando mudando a direção do seu olhar } \\
\text { assustado. }\end{array}$ \\
\hline
\end{tabular}

Fig. 18: Exemplo de traduções

Trevisan descreveu Madame Glynn "com a partitura aberta nas mãos", o que não corresponde à situação do original, que O'Shea preservou.

TradTerm, 15, 2009, p. 79-100 
Em outra ocasião, foi O'Shea que optou por não tentar reproduzir um discurso inventado de um personagem, criando uma frase inexistente no original, enquanto que Trevisan, com bastante sucesso, introduziu a mesma ideia do texto fonte:

\begin{tabular}{|l|l|}
\hline JJ & I'm a great fellow fol-the-diddle-I-do. \\
\hline HT & Sou muito importante, trá lá lá trá lá lá. \\
\hline OS & Por tudo o que fiz eu deveria exigir até mais. \\
\hline
\end{tabular}

Fig. 19: Exemplo de traduções

Problemas culturais induziram os tradutores a não usarem a ideia de uma lata de biscoitos de prata, pois culturalmente isso soaria muito estranho. Ambos optaram por introduzir o mesmo toque de sofisticação com a ideia de uma bandeja/salva de prata:

JJ She brought him into the drawing-room, made him sit down and brought out the decanter and the silver biscuit-barrel.

HT Ela conduziu-o à sala de visitas, convidou-o a se sentar e serviu bebida e biscoitos numa bandeja de prata.

os Mrs. Kearney conduziu-o à sala de visitas, convidou-o a sentar-se e serviu licor e doces numa salva de prata.

Fig. 20: Exemplo de traduções

Houve também um caso de apagamento de duplo sentido nas traduções da frase:

JJ When she came to the age of marriage she was sent out to many houses, where her playing and ivory manners were much admired.

HT Quando alcançou a idade de casar, foi enviada a muitas casas, onde seu talento e suas brilhantes maneiras eram muito admirados.

os Quando atingira a idade de se casar fizeram-na freqüentar diversas residências onde seus talentos musicais e suas maneiras se tornaram objeto de admiração.

Fig. 21: Exemplo de traduções

A expressão "ivory manners" evoca a ideia de "torre de marfim", do inatingivel, e também de frieza. "Brilhantes maneiras", ou simplesmente "maneiras" poderiam ter sido substituídas, a nosso

TRADTerm, 15, 2009, p. 79-100 
ver, por "maneiras de marfim", preservando a imagem criativa do autor. É também um caso de apagamento de uma figura de estilo, o que empobrece o texto literário.

Naturalmente, não cabe aqui uma análise exaustiva ou mesmo extensa dos desvios e possiveis problemas de tradução. Há questões de disparidades morfossintáticas e outras. Abordaremos apenas mais um aspecto do texto, interessante porque Joyce foi um autor muito cuidadoso com suas expressões e uma de suas preocupações era diferenciar o inglês irlandês, tanto no uso particular das palavras como sua pronúncia. Um personagem falando um inglês "flat", sem sotaque, é descrito negativamente, como alguém que não quer assumir posições nem denunciar origens. Assim, o tratamento dos sotaques é uma característica importante de Joyce, que foi até certo ponto apagada pelos tradutores, principalmente por Hamilton Trevisan:

JJ She noticed that he wore his soft brown hat carelessly on the side of his head and that his accent was flat.

HT Reparou que ele usava um chapéu mole, descuidadamente caído de lado, e que falava arrastado.

os Reparou que ele usava um chapéu marrom displicentemente caído de lado e que sua voz era monocórdia.

\begin{tabular}{|l|l}
\hline JJ & Mrs. Kearney rewarded his very flat final syllable with a quick
\end{tabular}

JJ stare of contempt, and then said to her daughter encouragingly:

HT A senhora Kearney acolheu a frase (cuja última palavra fora pronunciada com exagerada ênfase) com uma expressão de desprezo e disse encorajadora para a filha:

os Mrs. Kearney retribuiu-lhe com um olhar de desprezo a frase, cuja última sílaba fora pronunciada sem a menor expressão, e disse à filha, para animá-la:

Fig. 22: Exemplos de traduções

Na primeira frase a ausência de sotaque é equiparada com o fato de Fitzpatrick usar o chapéu "carelessly on the side of his head", uma maneira sutil de Joyce mostrar que o homem não tirou o chapéu diante de uma dama. Não fica claro, em qualquer das traduções, uma prosódia negativa, o que talvez pudesse ser introduzido de alguma maneira compensatória ou em alguma expressão do tipo "voz irritantemente sem sotaque".

TradTerm, 15, 2009, p. 79-100 


\section{Considerações Finais}

Pretendemos mostrar aqui algumas aplicações da Linguística de Corpus na análise de texto e no estudo de traduções. O programa WordSmith Tools dispõe, entre outras, de ferramentas que podem fornecer listas de palavras por ordem de frequência ou estatísticas. Nas comparações de corpora, obtivemos as palavras-chave, cuja relevância foi demonstrada, e também textos alinhados com suas traduções. Concordâncias, que são tão importantes na avaliação de contextos e prosódias, são ainda facilmente obtidas.

Evidentemente, esse tipo de análise não dispensa a observação minuciosa do pesquisador, o prévio estudo da obra literária envolvida e a própria microanálise textual, onde o julgamento do estudioso será imprescindível para determinar os aspectos relevantes da pesquisa. Acreditamos, porém, ter demonstrado o valor dessa metodologia, restando apenas ressaltar que a união da metodologia tradicional com o poderoso instrumental da Linguística de Corpus pode realmente dar uma nova dimensão à análise de textos literários, sejam eles traduzidos ou não.

\section{Referências bibliográficas}

DRABBLE, Margaret (ed.) (1996) The Oxford Companion to English Literature. Oxford: Oxford University Press, $5^{a}$ ed.

JOYCE, James (1994) Dublinenses. São Paulo: Siciliano, 2a ed. Tradução de José Roberto O'Shea.

(1996) Dubliners. London: Penguin Popular Classics. (1997) Dublinenses. Rio de Janeiro: Civilização Brasileira, $5^{\mathrm{a}}$ ed. Tradução de Hamilton Trevisan.

MAY, C.E. (ed.) (1994) The new short story theories. Athens: Ohio University Press.

SARDINHA, Tony Berber (2004) Lingüística de Corpus. São Paulo: Manole Ltda.

SCOTT, Mike (1998) WordSmith Tools (versão eletrônica). Oxford: Oxford University Press.

TradTerm, 15, 2009, p. 79-100 
100

\section{Sites eletrônicos:}

Textos de DUBLINERS: Disponivel em <http://gutenberg.net/dirs / etext01/dblnr11.txt>. Acesso e download em 02/09/04.

Textos de D. H. Lawrence: Disponiveis em <http://www.gutenberg net.au/ebooks03>. Acesso e download em 31/03/04.

Textos de K. Mansfield: Disponiveis em <http://www.gutenberg.net/ etext/1429>. Acesso e download em 31/03/04, e em <http://www.digital.library.upenn.edu/women/mansfield.html>. Acesso e download em 26/04/04.

Textos de V. Woolf: Disponíveis em <http://www.gutenberg.net.au/ ebooks02>. Acesso e download em 31/03/04.

Programa WordSmith Tools: Disponivel em <www.lexically.net/>.

TRADTERM, 15, 2009, p. 79-100 